\title{
Plant fertility defects induced by the enhanced expression of microRNA167
}

\author{
Peng $\mathrm{Ru}^{1}$, Lin $\mathrm{Xu}^{1}$, Hong $\mathrm{Ma}^{2,3}$, Hai Huang ${ }^{1,2}$ \\ ${ }^{l}$ National Laboratory of Plant Molecular Genetics, Shanghai Institute of Plant Physiology and Ecology, Shanghai Institute for Bio- \\ logical Sciences, 300 Fenglin Road, Shanghai 200032, China; ${ }^{2}$ SIBS-SJTU- PSU Joint Center for Life Sciences, Shanghai Institute of \\ Plant Physiology and Ecology, Shanghai Institute for Biological Sciences, 300 Fenglin Road, Shanghai 200032, China; ${ }^{3}$ Department \\ of Biology and the Huck Institute for the Life Sciences, The Pennsylvania State University, University Park, PA 16802, USA
}

The plant hormone auxin plays a critical role in regulating plant growth and development. Recent advances have been made in the understanding of auxin response pathways, primarily by the characterization of auxin response mutants in Arabidopsis. In addition, microRNAs (miRNAs) have been shown to be critical regulators of genes important for normal plant development and physiology. However, little is known about possible interactions between miRNAs and hormonal signaling during normal development. Here we show that an Arabidopsis microRNA, miR167, which has a complementary sequence to a portion of the AUXIN RESPONSE FACTOR6 (ARF6) and ARF8 mRNAs, can cause transcript degradation for $A R F 8$, but not for $A R F 6$. We report phenotypic characterizations of $35 S:: M I R 167 b$ transgenic lines, and show that severe $35 S:: M I R 167 \mathrm{~b}$ transgenic lines had phenotypes similar to those of an arf6 arf 8 double mutant. The transgenic phenotypes suggest that miR167 may repress $A R F 6$ at the level of translation. We demonstrate that the transgenic plants are defective in all four whorls of floral organs. In the transgenic flowers, filaments were abnormally short, anthers could not properly release pollen, and pollen grains did not germinate. Our results provide an important link between the miRNA-mediated regulatory pathway of gene expression and the auxin signaling network promoting plant reproductive development.

Cell Research (2006) 16:457-465. doi:10.1038/sj.cr.7310057; published online 15 May 2006

Keywords: Arabidopsis, auxin response factor, microRNA167, floral organ, pollen germination

\section{Introduction}

The plant hormone auxin plays a crucial role in developmental processes throughout the life cycle of a plant, including cell division and elongation, vascular tissue differentiation, root initiation, apical dominance, gravitropic and phototropic responses, fruit ripening, leaf senescence, and abscission of leaves and fruits [1]. How a simple chemical substance can affect such a wide variety of physiological processes has puzzled scientists in this field for many years. Recently, the identification of a gene family

Correspondence: Hai Huang

Tel: 21-64042090; Fax: 21-64042385;

E-mail: hhuang@iris.sipp.ac.cn

Received 18 Jan 2006; revised 8 Feb 2006; accepted 26 Feb 2006; published online 15 May 2006 encoding proteins called auxin response factors (ARFs) in Arabidopsis [2,3] provides a good entry to study auxin regulation at the molecular level. The ARF proteins can bind to the auxin-responsive cis elements in the promoter regions of members of an auxin-responsive gene family, GH3 [3]. The Arabidopsis genome encodes 23 known ARF proteins. Most ARF proteins contain an $\mathrm{N}$-terminal domain for DNA-binding, a central region, and a C-terminal domain for protein-protein interaction [4]. ARF proteins can either activate or repress downstream genes, primarily depending on the amino acid sequence of their central region [5].

Recently, miRNAs have been shown to play important roles in plant development and are widely believed to act as internal signals [6-9]. However, very little is known about the cross talk between miRNA signals and hormones. In Arabidopsis, a number of miRNAs were identified, showing critical roles in developmental regulations [7, 10-20]. 
Among the reported miRNA functions in Arabidopis, miR160 was found to regulate root cap formation by targeting ARF 10 and ARF 16, both of which control root cap cell differentiation [20]. The findings revealed a possible regulatory mechanism between miRNA and auxin and provide a system to study the interaction between miRNA signals and hormones, particularly the roles of ARFs, in plant development.

Recently, the functions of two of the $A R F$ genes, $A R F 6$ and $A R F 8$, have been shown by analyzing arf6 and arf8 mutant plants. Whereas the arf $8-1$ mutation only affects hypocotyl elongation in light conditions [21], the null mutants arf6-2 and arf8-3 showed slightly enhanced developmental abnormalities. Although both the floral organ number and position are normal in arf6-2 and arf8-3, the fertility in the single mutant plants is often reduced, and the double mutant is sterile [22]. Moreover, the sequence of a predicted miRNA, miR167, is complementary to a region of the ARF6 and ARF8 transcripts, within an encoding Cterminal AUXIN/IAA domain [23, 24]. However, it is not clear whether miR167 could play a role in downregulating these two $A R F$ genes. Here we report that miR167 can indeed reduce the level of $A R F 8$, but not $A R F 6$ transcripts, in transgenic plants that carried a $35 S:: M I R 167 b$ fusion. We show that the $35 S: \because M I R 167 b$ transgenic plants display altered morphology of all floral organs, resembling arf 8 single and arf6 arf 8 double mutants. These phenotypes of the transgenic plants suggest that unlike $A R F 8$, for which the mRNA level was reduced, ARF6 may be repressed translational. Our results indicate a possible regulatory role of miR167 in plant development. In addition, we find pollen germination in the $35 S: \because M I R 167 b$ transgenic plants is defective, indicating that $A R F 6$ and $A R F 8$ may play important roles in pollen germination.

\section{Materials and methods}

\section{Construction of a 35S::MIR167b fusion and plant growth}

A $185 \mathrm{bp}$ fragment for the MIR167b precursor was amplified using polymerase chain reaction (PCR) with genomic DNA from wild-type Col plants with gene-specific primers (5'-CAC AAC TTG TTG CTC AGG-3' and 5'-CAT GTG GAA TGC TTC ATC-3'). This DNA fragment was cloned into a T-vector (TaKaRa, Japan), verified by sequencing, and inserted into the plant transformation vector pMON530 (Monsanto, USA), downstream to the 35S promoter. This construct was introduced into Col plants by Agrobacterium-mediated transformation. The transgenic lines were verified by PCR, using a $35 S$-specific primer (5'-GCT CCT ACA AAT GCC ATC A-3') and a primer matching the MIR $167 \mathrm{~b}$ precursor sequence (5'-CAT GTG GAA TGC TTC ATC-3'). Plant growth was according to the conditions described previously [39].

\section{$R T-P C R$}

RNA extraction was according to our previous methods [40], with inflorescence from 20-day-old seedlings, and reverse transcription was performed with $1 \mu \mathrm{g}$ total RNA, using a kit (Fermentas, Vilnius, Lithuania). PCR was carried out with the following gene-specific primers: 5'-CAA AGG CAA AGG CAA ATC TCC C-3' and 5'-GAC AAC ACC TTC TAG TTG CAT AG-3' for ARF6, and 5'-CAT CAG GAG ATG CTG AAG CTT CC-3' and 5'-CGA GAGAGA TGC GAA CGA ATG GC-3' for $A R F 8$.

\section{MicroRNA filter hybridization}

Total RNA was extracted as described previously [41], with inflorescences of 3-week-old plants. miRNA filter hybridization was according to our previous methods [7], with a miR167 antisense probe, 5'-TAG ATC ATG CTG GCA GCT TCA-3'.

\section{Microscopy}

Fresh tissues of wild-type and overexpression plants were examined using an SZH10 dissecting microscope, and photographed using a Nikon E995 digital camera (Nikon, Japan). The method for SEM was described previously [39]. For pollen observation, stamens were incubated in $1 \%$ Lugol for $3 \mathrm{~min}$ and were mounted on the glass slide in $75 \%$ chloralhydrate, before viewing [42].

\section{Pollen germination}

Pollen was isolated form the same-stage anthers of wild-type and $35 S:: M I R 172 b$ transgenic plants and was germinated in medium containing $0.36 \mathrm{mg} / \mathrm{ml} \mathrm{CaCl}, 0.08 \mathrm{mg} / \mathrm{ml} \mathrm{H}_{3} \mathrm{BO}_{3}, 20 \%$ sucrose, $0.01 \mathrm{mg} / \mathrm{ml}$ myo-inositol, $1 \%$ gelatin, and $1 \%$ noble agar. Glass slides were dipped into warm unsolidified medium, and then cooled to form a thin layer on the slide. Pollen was tapped onto these slides and germinated in a humid chamber for 8-16 $\mathrm{h}$ [43].

\section{Results}

\section{Generation of $35 S:: M I R 167 b$ transgenic plants}

The Arabidopsis genome contains two copies of miR167, MIR 167a, and MIR167b (Figure 1A), which are located at different loci [24]. Although sequences of the two precursors (pre-miR167 s) are different, these contain the same mature miR167 sequence. To study miR167 functions, we cloned the pre-miR $167 \mathrm{~b}$ sequence, consisting of 21 nucleotide (nt) of mature miR167 flanked by 40 and 36 nt 5 ' and 3 ' sequences, respectively. After verification by sequencing, the pre-miR167b fragment was inserted into a plant transformation vector (pMON530), downstream of the constitutive $35 \mathrm{~S}$ promoter (Figure 1E). This construct was then introduced into Arabidopsis ecotype Columbia (Col) by Agrobacterium-mediated transformation.

A total of 117 independent transgenic lines were obtained and a subset of these transgenic plants were examined for the presence of the transgene, with primers corresponding to the sequences of the $35 \mathrm{~S}$ promoter and pre-miR167 (Figure 1E). T-DNA insertions containing 35S::MIR167b were detected in all transgenic plants analyzed (Figure 1F). Among the transgenic plants, 38 lines displayed severe phenotypes with a smaller plant (Figure 1C), in comparison with wild-type plants (Figure 1B). In addition, 36 other lines had less severe phenotypes (Figure 1D), but were 


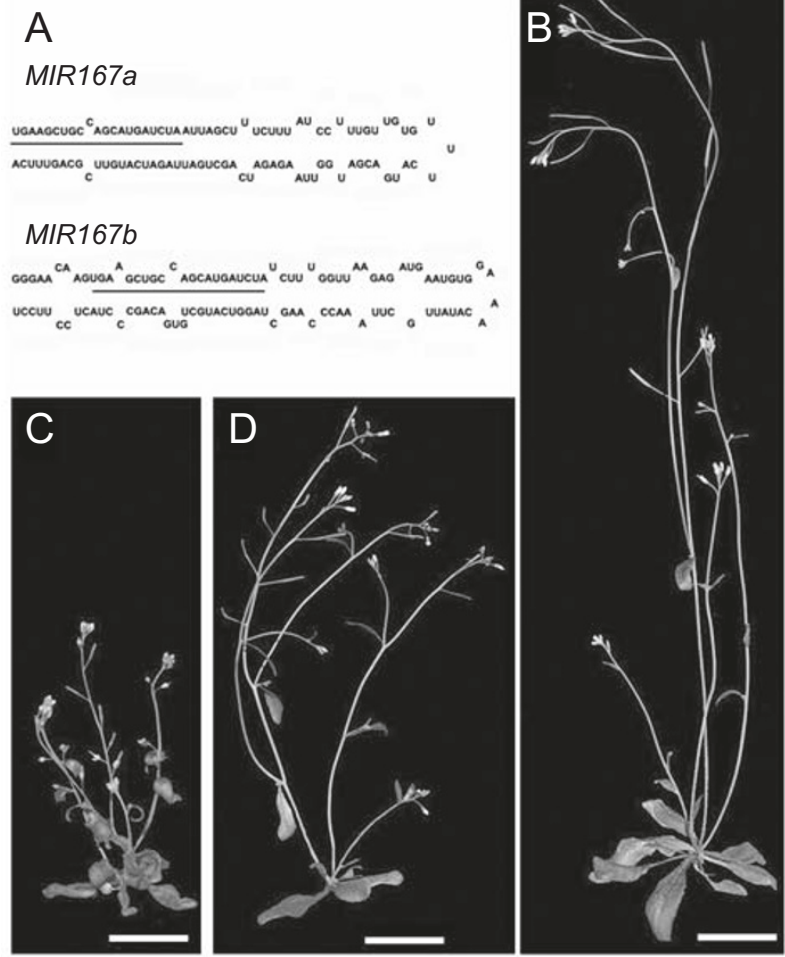

E

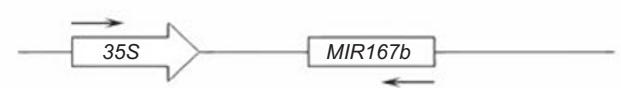

$\mathrm{F}$
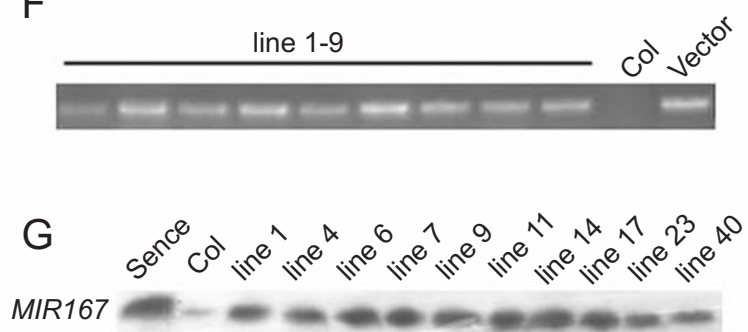

5S rRNA

Figure 1 Characterization of the $35 S:: M I R 167 b$ transgenic plants. (A) Pre-miR167 sequences, miR167a (upper panel) and miR167b (lower panel). Mature miR167 sequences are underlined. (B-D) Plant statures of wild-type Col (B), a group I transgenic plant line-14 (C), and a group II transgenic plant line-17 (D). Bars $=1 \mathrm{~mm}$ in (B) to (C). (E) The structure of $35 S:: M I R 167 b$ fusion. Arrows indicate positions of the primers used in detecting the presence of the $35 S: \because M I R 167 b$ transgene. (F) Identification of $35 S:: M I R 167 b$ transgenic plants by PCR. PCR products were from DNAs of nine independent T1 transgenic plants. (G) MicroRNA blot hybridization using a miR167 antisense probe. Among the RNA samples examined, lines 1, 4, 6, $7,9,11$, and 14 belong to group I transgenic plants, whereas lines 17,23 , and 40 are group II plants. Note that all examined transgenic lines contained over-accumulated miR167, as compared to that in the wild-type plants. clearly shorter than the wild-type plant. We refer to the 38 severe lines and 36 less severe lines as group I and II transgenic plants, respectively. We also obtained 32 lines with phenotypes close to those of the wild-type plant (data not shown), and 11 lines showing different phenotypes possibly unrelated to miRNA167 activities (data not shown). To determine whether the transgenic lines had over-accumulated miR167, we performed RNA blot hybridization using RNA samples from ten independent lines. Our results revealed that the pre-miR 167 sequence driven by the $35 \mathrm{~S}$ promoter can be properly processed into mature miR 167 , resulting in a much higher miR167 level than that in the wild type (Figure 1G).

\section{S::MIR167b transgenic plants have the reduced ARF8 transcript level}

To determine whether the increased miR167 level could cause degradation of transcripts from $A R F 6$ and/or $A R F 8$, we analyzed mRNA levels of these two genes using RT-PCR. The miR167 sequence is complementary to the ARF6 and ARF8 mRNAs that encode a portion of the conserved $\mathrm{C}$-terminal domain of the proteins, but with four and three mismatches for the $A R F 6$ and the $A R F 8$ pairing regions, respectively, among the 21 nts (Figure 2A). Our RT-PCR results from six independent transgenic lines showed that the levels of $A R F 8$ transcripts were decreased markedly compared to that in the wild type, whereas the ARF6 levels were almost unchanged (Figure 2B). These results strongly support the hypothesis that the $A R F 8$, but not $A R F 6$, mRNAs were degraded in the $35 S:: M I R 167 b$ transgenic plants.

\section{Phenotypic analyses of the $35 S: \because M I R 167 b$ transgenic plants}

To understand better the role of miR167 and functions of $A R F 6$ and $A R F 8$ in plant development, we performed phenotypic characterizations of the group I and II $35 S:$ : $M I R 167 b$ transgenic plants. In addition to the shorter plant statures of $35 S:: M I R 167 b$ plants (Figures 1B-1D), group I plants produced completely sterile flowers, although the group II flowers were barely fertile. Compared to the wildtype inflorescence (Figure 3A, left), those of the group I (Figure 3A, middle) and the group II (Figure 3A, right) lines appeared more compacted, with short internodes between flowers and a short pedicel for each flower. Sepals and petals of the $35 S: \because M I R 167 b$ flowers always tightly surrounded the gynoecium and barely detached even after the siliques had fully elongated (Figure 3B). Sepals (Figure 3C) and petals (Figure 3D) were usually smaller in size in the $35 \mathrm{~S}:$ : MIR167b plants than those in the wild type. Filaments of the group I stamens were very short (Figure 3E, middle), although filaments of the group II plants (Figure 3E, left) 
A

MIR167 3' ... AUC UAG UAC GAC CGU CGA AGU ... 5'

ARF6 $55^{\prime} . .$. a AG ATC A gG CTG GCA GCT T gt ... 3'

ARF8 $55^{\prime}$... TAG ATC A gG CTG GCA GCT T gt ... 3'

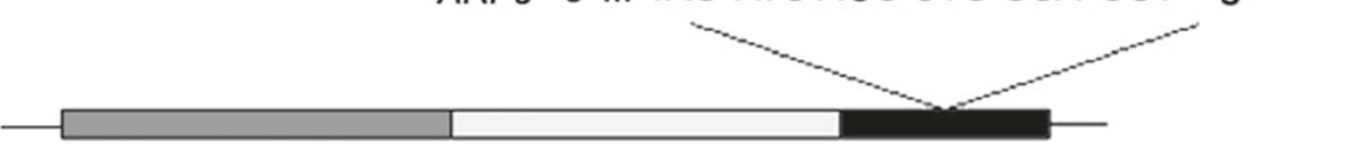

DNA binding domain $\quad$ Q rich domain $\quad$ AUX/IAA domain

B

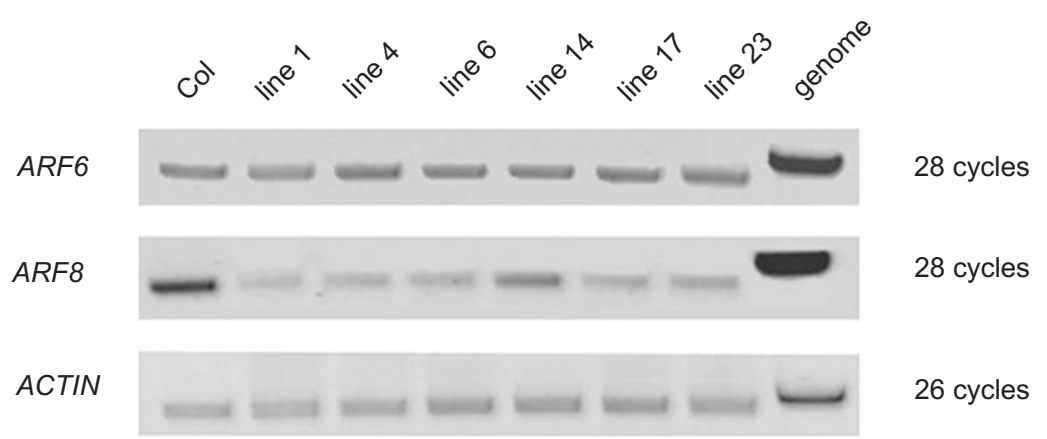

Figure 2 RT-PCR analyses of $A R F 6$ and $A R F 8$ expression. (A) The miR167 sequence, matching a region of the $A R F 6$ and $A R F 8$ mRNA encoding in the AUX/IAA domain. The letters in lower case indicate mismatches between miR167 and $A R F 6 / A R F 8$ sequences. (B) RT-PCR of $A R F 6$ and $A R F 8$ using inflorescence of the T1 $35 S:: M I R 167 b$ transgenic lines. Note that $A R F 6$ transcript levels were unchanged, whereas the $A R F 8$ levels were reduced markedly.
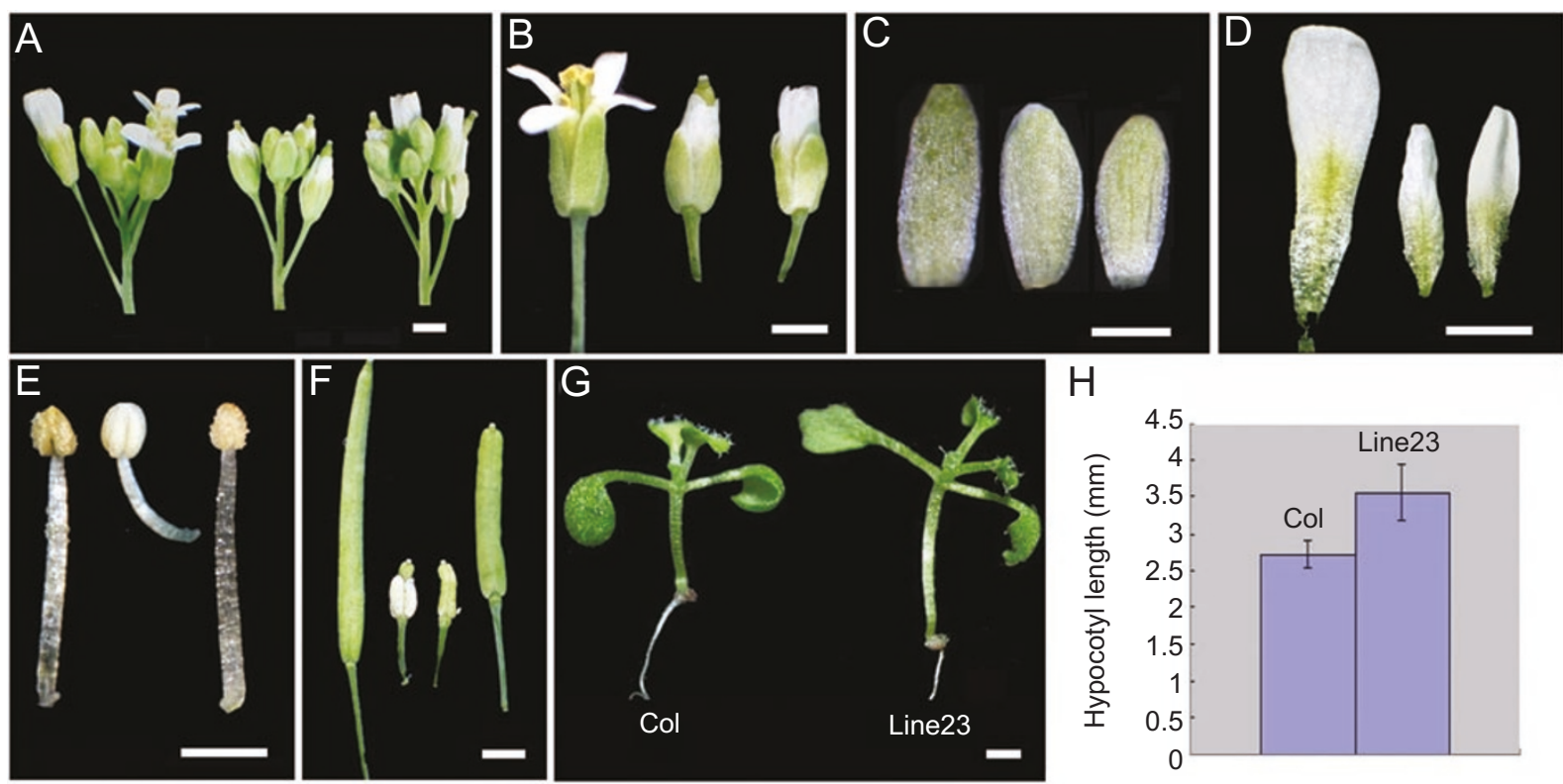

Figure 3 Phenotypes of flower organs of the $35: \because M I R 167 b$ transgenic lines. All floral organs are arranged in the order of Col (left), the group I plant line-14 (center) and the group II plant line-17 (right), except where otherwise indicated. (A) Inflorescence. (B) Flowers. (C) Sepals. (D) Petals. (E) Stamens. (F) Siliques. All group I plants produced siliques with a very small size, and sepals and petals were consistently attached to the siliques even at a stage when the wild-type seeds have matured. Two line-14 siliques are shown, with sepals and petals removed for the right one. (G) Seedlings of 8-day-old wild-type and a T2 group II plant (line-23), showing an elongated hypocotyl in the transgenic plants. Bars $=1 \mathrm{~mm}$ in (A), (B), (E), (F), and (G), and $0.5 \mathrm{~mm}$ in (C) and (D). (H) Measurement of hypocotyl length using 8-day-old seedlings grown under continuous white light at $22{ }^{\circ} \mathrm{C}$. A total of 55 wild-type and 35 T2 line-23 plants were scored. Bars indicate standard error. 
were similar in length to that of the wild type (Figure 3E, right). In addition, the anther color of the group I plants were buff, different from that of the wild-type anthers, and all group I anthers did not release pollens in their maturations (Figure 3E, middle). Compared to the wild type (Figure 3F, left), the infertile gynoecia of the group I plants were very small (Figure 3F, middle). Although the gynoecia of group II plants had a few seeds set, these were also shorter in length (Figure 3F, right) than those in wild-type plants.

It was reported previously that arf8- 1 mutation resulted in seedlings with a long hypocotyl in the light [21]. To test whether the 35S:MIR167b transgene also affected hypocotyl length, we examined hypocotyl length of the T2 seedlings of the group II lines, and found that they had longer hypocotyls than wild-type seedlings (Figure $3 \mathrm{G}$ and $3 \mathrm{H})$. This phenotype resembles that in the arf8-1 single mutant [21], and other phenotypes described above are very similar to those of the arf6-2 afr 8-3 double mutant plants [22]. Therefore, it is possible that both $A R F 6$ and $A R F 8$ are repressed in the $35 S:: M I R 167 b$ transgenic plants.

\section{The cellular pattern of floral organs in the $35 S:: M I R 167 b$ plants}

The smaller floral organs in the $35 S: \because M I R 167 b$ transgenic plants suggest a defect either in cell division or in cell elongation. To understand phenotypic alterations of the $35 S:: M I R 167 b$ transgenic plants at a cellular level, we analyzed floral organs of group I plants using scanning electron microscopy (SEM). In comparison to the wild type (Figure 4A-4C), petal epidermal cells of the group I plants were less elongated (Figure 4E and 4F); the similar situation was observed on the filament, where wild-type epidermal cells (Figure $4 \mathrm{G}$ and $4 \mathrm{H}$ ) were much longer than those on filaments of the group I transgenic plants (Figure $4 \mathrm{~J}$ and $5 \mathrm{~K}$ ). Compared to the wild-type anther (Figure 4I),
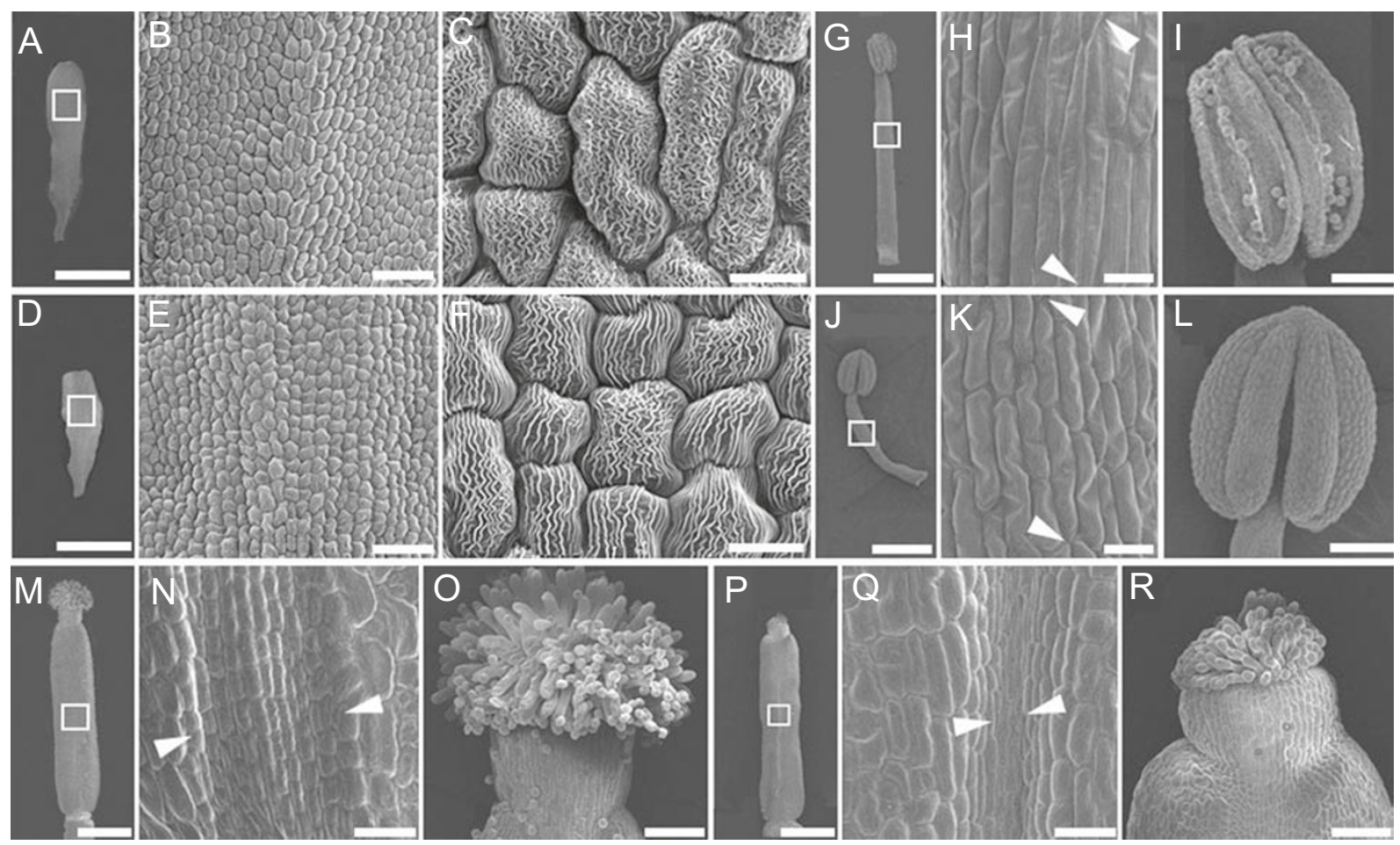

Figure 4 SEM of floral organs. (A-F) Petals of 3-week-old wild-type (A-C) and the T1 group I plants (line-6) (D-F). (A) Wild type. (B) A close-up of the boxed region in (A). (C) Further magnification of the epidermal cells in (B). (D) Line-6. (E) A close-up of the boxed region in (D). (F) Further magnification of the epidermal cells in (E). (G) A wild-type stamen. (H) Filamentous epidermal cells of the wild type. (I) A mature wild-type anther with pollen released. (J) A line-6 stamen. (K) Filamentous epidermal cells of line-6. (L) A line-6 anther being indehiscent at the same stage as that in (I). (M-R) Gynoecium phenotypes of wild type and group-I $35::$ MIR167b transgenic plants. (M) A wild-type gynoecium. (N) A close-up of the boxed replum region in (M). (O) A close-up of the style in (M). (P) A line-6 gynoecium. (Q) A close-up of the boxed replum region in (P). (R) A close-up of the style in (P). Bars $=1 \mathrm{~mm}$ in $\mathbf{( A )}$ and $(\mathbf{D}) ; 50 \mu \mathrm{m}$ in $\mathbf{( B )}$ and $(\mathbf{E}) ; 10 \mu \mathrm{m}$ in $(\mathbf{C})$ and $(\mathbf{F}) ; 0.5 \mathrm{~mm}$ in $(\mathbf{G}),(\mathbf{J}),(\mathbf{M})$, and $(\mathbf{P}) ; 20 \mu \mathrm{m}$ in $(\mathbf{H}),(\mathbf{K}),(\mathbf{N})$, and (Q); $0.2 \mathrm{~mm}$ in (I) and (L); and $100 \mu \mathrm{m}$ in (O) and (Q). 
the anther of group I plants were always closed (Figure $4 \mathrm{~L})$. Interestingly, the replum of the group I plants were deeply dented (Figure 4P and 4Q) as compared to that in the wild-type plants (Figure $4 \mathrm{M}$ and $4 \mathrm{~N}$ ). Similar to the short petal and filament cells, the stigma cells of the group I plants were also very short (Figure 4R), in comparison to those in the wild-type plants (Figure 4O). All these results indicate that the $A R F 6$ and $A R F 8$ functions are required for cell elongation in several floral organs.

Pollen germination is defective in the $35 S:: M I R 167 b$ transgenic plants

As described above, group I $35 S:: M I R 167 b$ transgenic plants produced stamens with a short filament (Figure 4J) and an undehisced anther (Figure $4 \mathrm{~L}$ ). These phenotypes provide explanations for the reduced fertility in the $35 S:$ : MIR 167b transgenic plants [22]. In addition, we observed, by potassium iodine staining, that both group I and II anthers produced normal-shaped pollen grains that contained starch (Figure 5B and 5C), similar to those in the wild-type anther (Figure 5A). To determine whether pollen grains of the $35 S:: M I R 167 b$ anther were functionally defective, we first performed reciprocal crosses between wild-type and $35 S: \because M I R 167 b$ plants. Our results showed that the $35 S::$ $M I R 167 b$ gynoecia that were pollinated with the wild-type pollen produced seeds, although the resulted siliques were shorter than normal. On the contrary, when wild-type flowers were used as the female and pollinated with the pollen released from anthers of the $35 S:: M I R 167 b$ plant, no seeds were produced (Table 1). This result indicates that pollen defects likely contributed to the reduced fertility in $35 S:$ : MIR167b plants.

To further verify whether pollen grains in the $35 S:$ : MIR $167 b$ anthers were fertile, we performed pollen germination experiments. A severe transgenic line (line-14) and a less severe transgenic line (line-17), representing group I and II $35 S:: M I R 167 b$ transgenic plants, respectively, were analyzed in this experiment. As compared to the wild-type

Table 1 Fertility tests by reciprocal crosses between wild-type and $35 S:: M I R 167 b$ transgenic plants

\begin{tabular}{llllll}
\hline Crosses & Female & Male & $\begin{array}{l}\text { Fertile } \\
\text { siliques }\end{array}$ & $\begin{array}{l}\text { Sterile } \\
\text { siliques }\end{array}$ & $\begin{array}{l}\text { Total }^{\mathrm{a}} \\
\text { flowers }\end{array}$ \\
\hline \multirow{2}{*}{ Group I } & Line-4 & Wild type & 3 & 0 & 3 \\
& Line-11 & Wild type & 2 & 0 & 2 \\
& wild type & Lines 4/11 & 0 & 7 & 7 \\
Group II & Line-23 & Wild type & 2 & 0 & 2 \\
& Line-40 & Wild type & 3 & 0 & 3 \\
\hline
\end{tabular}

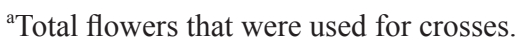

${ }^{b}$ Both line- 4 and line- 11 were used in pollination.
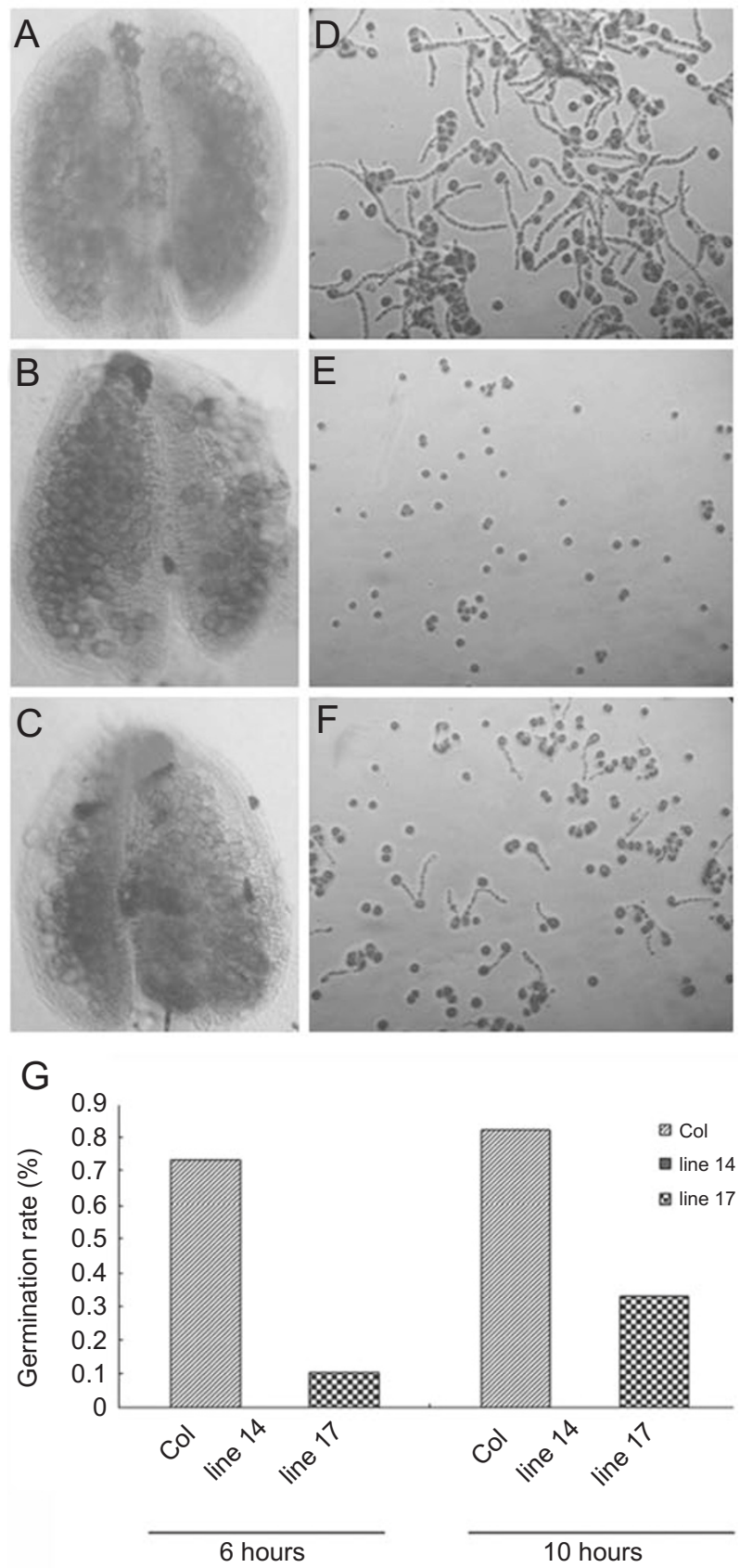

Figure 5 Starch color assay and pollen germination. (A-C) Starch assay of the pollen in the wild type (A), a group I transgenic plant line-14 (B), and a group II transgenic plant line-23 (C). (D-F) Germination of the wild type (D), line-14 (E), and line-17 (F) pollen grains, which were germinated on a medium for $6 \mathrm{~h}$. (F) Measurement of pollen germination rate of the wild type, line-14 and line-17 at the $6^{\text {th }}$ and $10^{\text {th }} \mathrm{h}$, respectively, after pollen grains were germinated on the medium.

pollen (Figure 5D and 5G), the germination rate of pollen from line- 17 was dramatically reduced (Figure 5F and 5G). More dramatically, germinated pollen grains were not ob- 
served among the pollen from line-14 (Figure 5E and 5G). These results indicate that the pollen germination defect is also an important factor leading to the reduced fertility or sterility in the $35 S:: M I R 167 b$ transgenic plants.

\section{Discussion}

The Arabidopsis genome contains 23 ARF genes, and different ARF proteins can form homodimers and heterodimers in response to the auxin signal [4]. ARF proteins are known to act upstream of many physiological effects of auxin. Although a few $A R F$ genes have been characterized functionally [21, 22, 25-29], the functions for many other $A R F s$ remain unknown. Recent studies indicate that the several $A R F$ genes are negatively regulated via small RNAs at the post-transcriptional level. For example, $A R F 2, A R F 3$, and $A R F 4$ are repressed by trans-acting siRNA [30-33]; and $A R F 10, A R F 16$, and $A R F 17$ are regulated by miR 160 [20]. In this study, we show that overexpression of miR167, which matches in sequence to the ARF6 and ARF8 coding region, resulted in phenotypes similar to those of the arf6 arf 8 double mutant. These results strongly suggest a likely regulatory relationship between miR167 and ARF6/ARF8. These specific instances highlight a possible general regulation of $A R F$ expression by miRNAs, indicating an importance for small RNAs in auxin signaling responses.

Both the reduced levels of the ARF 8 mRNA and the seedling and floral phenotypes of the $35 S:: M I R 167 \mathrm{~b}$ transgenic plants strongly support the hypothesis that the increased miR167 level resulted in greater extent of miR167-mediated cleavage of the $A R F 8$ transcript. On the other hand, the ARF6 mRNA level was not reduced in the $35 S: \because M I R 167 b$ transgenic plants. Nevertheless, the $35 S:: M I R 167 b$ transgenic plants exhibited strong floral phenotypes found only in the arfo arf 8 double mutant, not in the arf8 single mutant. Therefore, it is likely that the $A R F 6$ function is also negatively regulated by the $35 S::$ $M I R 167 b$ transgene.

It is known that miRNA can target mRNAs for either cleavage or translational repression [34]. The repression of protein synthesis by miRNAs is a common mechanism in animals [35, 36], and has also been found in plants [34]. For example, the Arabidopsis APETALA2 (AP2) gene encodes a transcription factor that regulates flower development, and a miRNA, miR172, is known to pair to the $A P 2$ transcript to repress the protein translation $[10,11]$. Although the level of $A P 2$ transcripts remains unchanged in the $35 S:: M I R 172$ transgenic lines, the AP2 protein level was dramatically reduced. Therefore, it is possible that miR167-mediated repression of $A R F 6$ function is at the level of translation. The alignments of miR 167 with $A R F 6$ and $A R F 8$ contain four and three nt mismatches, respectively. It is known that the degree of miRNA-target sequence matches greatly influences whether the target mRNA is cleaved. In Arabidopsis, for example, the miR165 and miR166 sequences match a PHAVOLUTA (PHV) region by 18 and $17 \mathrm{nt}$, respectively. Although both miR165 and miR166 can cause $P H V$ transcript cleavage, miR165 supports a higher cleavage activity than miR166 [14]. Therefore, the observation that ARF6 level was not detectably reduced in the $35 S:: M I R 167 b$ transgenic plants may be explained by the fact that fewer base pairs between ARF6 and miRNA167 may result in inefficient cleavage of the $A R F 6$ transcripts.

Our results also revealed that overexpression of the premiR167 sequences resulted in the altered floral phenotypes in the $35 S:: M I R 167 b$ transgenic plants, although the leaf phenotypes kept unchanged (data not shown). All floral organs of the transgenic plants were reduced in size and the flowers were sterile, similar to those in the arf6 arf8 double mutant [22]. It was reported that auxin is crucial for flower induction $[37,38]$. Loss of function in PIN1, which encodes an Arabidopsis auxin transporter, results in plant inflorescence without any floral primordium, whereas addition of auxin to the inflorescence tip could rescue this phenotype [38]. Our results suggest that auxin may be involved in the regulation of floral organ development. We further performed detailed phenotypic analyses and found that the transgenic plants and the arf6 arf 8 double mutant both produced short filaments and indehiscent anthers, which contribute to the fertility defects. In addition, we observed some novel phenotypes in the present study that were not reported in the previous characterizations of arf6 and arf 8 single, and afr6 arf 8 double mutants. First, cell elongation in the floral organs of transgenic plants, such as petals and filaments, were aberrant. Secondly, pollen germination rate of the transgenic plants was dramatically reduced. The abnormal pollen germination in the $35 S:$ : MIR $167 b$ transgenic plants is another important factor that contributes to the male sterility, providing strong evidence for another $A R F 6$ and $A R F 8$ function, and a possible auxin action, in regulating reproductive development.

\section{Acknowledgments}

The authors thank X Gao for assistance with the SEM analysis, L Pi for some experimental protocols, and L Yang for discussion of this work. This research was supported by grants from Shanghai Institutes for Biological Sciences for the Plant Reproductive Development to $\mathrm{H} \mathrm{Ma}$ and from the Shanghai Scientific Committee to H Huang.

\section{References}

1 Eckardt NA. New Insights into Auxin Biosynthesis. Plant Cell 2001; 13:101-111. 
2 Kim J, Harter K, Theologis A. Protein-protein interactions among the Aux/IAA proteins. Proc Natl Acad Sci USA 1997; 94:1178611791.

3 Ulmasov T, Murfett J, Hagen G, Guilfoyle TJ. Aux/lAA proteins repress expression of reporter genes containing natural and highly active synthetic auxin response elements. Plant Cell 1997; 9:1963-1971.

4 Liscum E, Reed JW. Genetics of Aux/IAA and ARF action in plant growth and development. Plant Mol Biol 2002; 49:387400.

5 Tiwari SB, Wang XJ, Hagen G, Guilfoyle GT. AUX/IAA proteins are active repressors, and their stability and activity are modulated by auxin. Plant Cell 2001; 13:2809-2822.

6 Llave C, Kasschau KD, Rector MA, Carrington JC. Endogenous and silencing-associated small RNAs in plants. Plant Cell 2002; 14:1605-1619.

7 Li H, Xu L, Wang H, et al. The Putative RNA-dependent RNA polymerase RDR6 acts synergistically with ASYMMETRIC LEAVES 1 and 2 to repress BREVIPEDICELLUS and MicroRNA165/166 in Arabidopsis leaf development. Plant Cell 2005; 17:2157-2171.

8 Juarez MT, Kui JS, Thomas J, Heller BA, Timmermans MC. microRNA-mediated repression of rolled leafl specifies maize leaf polarity. Nature 2004; 428:84-88.

9 Carrington JC, Ambros V. Role of microRNAs in plant and animal development. Science 2003; 18:336-338.

10 Aukerman MJ, Sakai H. Regulation of flowering time and floral organ identity by a MicroRNA and its APETALA2-like target genes. Plant Cell 2003; 15(11):2730-41.

11 Chen XM. A microRNA as a translational repressor of APETALA2 in Arabidopsis flower development. Science 2004; 303:2022-2025.

12 Palatnik JF, Allen E, Wu X, et al. Control of leaf morphogenesis by microRNAs. Nature 2003; 425:257-263.

13 Emery JF, Floyd SK, Alvarez J, et al. Radial patterning of Arabidopsis shoots by class III HD-ZIP and KANADI genes. Curr Biol 2003; 13:1768-1774.

14 Tang G, Reinhart BJ, Bartel DP, Zamore PD. A biochemical framework for RNA silencing in plants. Genes Dev 2003; 17:4963.

15 Timmermans MC, Juarez MT, Phelps-Durr TL. A conserved microRNA signal specifies leaf polarity. Cold Spring Harb Symp Quant Biol 2004; 69:409-417.

16 Kidner CA, Martienssen RA. Spatially restricted microRNA directs leaf polarity through ARGONAUTE1. Nature 2004; 428:81-84.

17 Mallory AC, Dugas DV, Bartel DP, Bartel B. MicroRNA regulation of NAC-domain targets is required for proper formation and separation of adjacent embryonic, vegetative, and floral organs. Curr Biol 2004; 14:1035-1046.

18 Mallory AC, Reinhart BJ, Jones-Rhoades MW, et al. MicroRNA control of PHABULOSA in leaf development: importance of pairing to the microRNA 5' region. EMBO J 2004; 23:33563364.

19 Achard P, Herr A, Baulcombe DC, Harberd NP. Modulation of floral development by a gibberellin-regulated microRNA. Development 2004; 131:3357-3365.

20 Wang JW, Wang LJ, Mao YB, et al. Control of root cap formation by MicroRNA-targeted auxin response factors in Arabidopsis.
Plant Cell 2005; 17:2204-2216.

21 Tian CE, Muto H, Higuchi K, et al. Disruption and overexpression of auxin response factor 8 gene of Arabidopsis affect hypocotyl elongation and root growth habit, indicating its possible involvement in auxin homeostasis in light condition. Plant J 2004; 40:333-343.

22 Nagpal P, Ellis CM, Weber H, et al. Auxin response factors ARF6 and ARF8 promote jasmonic acid production and flower maturation. Development 2005; 132:4107-4118.

23 Rhoades MW, Reinhart BJ, Lim LP, Burge CB, Bartel B, Bartel DP. Prediction of plant microRNA targets. Cell 2002; 110:513520.

24 Reinhart BJ, Weinstein EG, Rhoades MW, Bartel B, Bartel DP. MicroRNAs in plants. Genes Dev 2002; 16:1616-1626.

25 Hardtke CS, Berleth T. The Arabidopsis gene MONOPTEROS encodes a transcription factor mediating embryo axis formation and vascular development. EMBO J 1998; 17:1405-1411.

26 Harper RM, Stowe-Evans EL, Luesse DR, et al. The NPH4 locus encodes the auxin response factor ARF7, a conditional regulator of differential growth in aerial Arabidopsis tissue. Plant Cell 2000; 12:757-770.

27 Nemhauser JL, Feldman LJ, Zambryski PC, Auxin and ETTIN in Arabidopsis gynoecium morphogenesis. Development 2000; 127:3877-3888.

28 Okushima Y, Mitina I, Quach HL, Theologis A. AUXIN RESPONSE FACTOR 2 (ARF2): a pleiotropic developmental regulator. Plant J 2005; 43:29-46.

29 Okushima Y, Overvoorde PJ, Arima K, et al. Functional genomic analysis of the AUXIN RESPONSE FACTOR gene family members in Arabidopsis thaliana: unique and overlapping functions of ARF7 and ARF19. Plant Cell 2005; 17:444-463.

30 Peragine A, Yoshikawa M, Wu G, Albrecht HL, Poethig RS. SGS3 and SGS2/SDE1/RDR6 are required for juvenile development and the production of trans-acting siRNAs in Arabidopsis. Genes Dev 2004; 18:2368-2379.

31 Allen E, Xie Z, Gustafson AM, Carrington JC. microRNA-directed phasing during trans-acting siRNA biogenesis in plants. Cell 2005; 121:207-221.

32 Yoshikawa M, Peragine A, Park MY, Poethig RS. A pathway for the biogenesis of trans-acting siRNAs in Arabidopsis. Genes Dev 2005; 19:2164-2175.

33 Williams L, Carles CC, Osmont KS, Fletcher JC. A database analysis method identifies an endogenous trans-acting shortinterfering RNA that targets the Arabidopsis ARF2, ARF3, and ARF4 genes. Proc Natl Acad Sci USA 2005; 102:9703-9708.

34 Dugas DV, Bartel B. MicroRNA regulation of gene expression in plants. Curr Opin Plant Biol 2004; 7:512-520.

35 Wightman B, Ha I, Ruvkun G. Posttranscriptional regulation of the heterochronic gene lin-14 by lin- 4 mediates temporal pattern formation in C. elegans. Cell 1993; 75:855-862.

36 Couzin J. Breakthrough of the year. Small RNAs make big splash. Science 2002; 298:2296-2297.

37 Okada K, Ueda J, Komaki MK, Bell CJ, Shimura Y. Requirement of the auxin polar transport system in early stages of Arabidopsis floral bud formation. Plant Cell 1991; 3:677-684.

38 Reinhardt D, Mandel T, Kuhlemeier C. Auxin regulates the initiation and radial position of plant lateral organs. Plant Cell 2000; 12:507-518.

39 Chen C, Wang S, Huang H. LEUNIG has multiple functions in 
gynoecium development in Arabidopsis. Genesis 2000; 26:4254.

$40 \mathrm{Xu} \mathrm{L}, \mathrm{Xu}$ Y, Dong A, et al. Novel as1 and as2 defects in leaf adaxial-abaxial polarity reveal the requirement for ASYMMETRIC LEAVES1 and 2 and ERECTA functions in specifying leaf adaxial identity. Development 2003; 130:4097-4107.

41 Huang H, Tudor M, Weiss CA, Hu Y, Ma H. The Arabidopsis MADS-box gene AGL3 is widely expressed and encodes a sequence-specific DNA-binding protein. Plant Mol Biol 1995; 28:549-567.
42 van den Berg C, Willemsen V, Hendriks G, Weisbeek P, Scheres B. Short-range control of cell differentiation in the Arabidopsis root meristem. Nature 1997; 390:287-289.

43 Fan LM, Wang YF, Wang H, Wu WH. In vitro Arabidopsis pollen germination and characterization of the inward potassium currents in Arabidopsis pollen grain protoplasts. J Exp BotJ 2001; 52:1603-1614.

Edited by Sheng Luan 